\title{
COVID-19 Pandemic in India: A Mathematical Model
}

\section{Study}

Sudhanshu Kumar Biswas ${ }^{1}$, Jayanta Kumar Ghosh ${ }^{2}$, Susmita Sarkar ${ }^{3}$, and Uttam

\author{
Ghosh $^{* 3}$
}

${ }^{1}$ Department of Mathematics, Sripat Singh College, Murshidabad, West Bengal, India.

${ }^{2}$ Boalia Junior High School, Nadia, West Bengal, India.

${ }^{3}$ Department of Applied Mathematics, University of Calcutta, Kolkata, India.

May 30, 2020

\begin{abstract}
:
The present novel corona virus $(2019-\mathrm{nCoV})$ infection has created a global emergency situation by spreading all over the world in a large scale within very short time period. The infection induced death rate is also very high. There is no vaccine or anti-viral medicine for such infection. So at this moment a major worldwide problem is that how we can control this pandemic. On the other hand, India is a high population density country, where the corona virus disease (COVID-19) has started to spread from $1^{s t}$ week of March, 2020 in a significant number of COVID-19 positive cases. Due to this high population density human to human social contact rate is very high in India. So control of the pandemic COVID-19 in early stage is very urgent and challenging problem. Mathematical models are employed in this paper to study the COVID-19 dynamics, to identify the influential parameters and to find the proper prevention strategies to reduce the outbreak size. In this work, we have formulated a deterministic compartmental model to study the spreading of COVID-19 and estimated the model parameters by fitting the model with reported data of ongoing pandemic in India. Sensitivity analysis has been done to identify the key

*Corresponding author Email:uttam_math@yahoo.co.in
\end{abstract}


model parameters. The basic reproduction number has been estimated from actual data and the effective basic reproduction number has been studied on the basis of reported cases. Some effective preventive measures and their impacts on the disease dynamics have also been studied. Future trends of the disease transmission has been Predicted from our model with some control measures. Finally, the positive measures to control the disease have been summarized.

Keywords: Basic reproduction number, COVID-19, Asymptomatic class, Quarantine, Sensitivity analysis, Preventive measures.

AMS Subject Classification 2010: 37N25, 49J15, 92D30

\section{Introduction}

The corona virus disease (COVID-19) is a worldwide infectious disease in the current time [1, 2, 3, 4, 5]. Including this year 2020, the world faces severe attack by corona virus several times, since then: (i) SARS-CoV occurred in China and then spread many neighbouring countries in 2002-2003 [6], (ii) MERS-CoV appeared in 2012 throughout the Middle East [7, 8, 9, 10]. The third one is SARS-CoV-2 which is first identified in China in December 2019 and the whole world has been suffering from it [11]. The symptoms of all corona virus patients are almost same, they suffer with respiratory problem but COVID-19 is more infectious compared to its predecessors[12]. Most of the countries of the World are affected by this disease and its harmfulness is increasing day by day. The novel corona virus was first identified by health authority of Wuhan province of China[13, 14, 15, 16] . Then it spreaded to different countries mainly through air-travel mode as large number of people are associated with this state $[17,18,19]$. To control the disease spreading, the World Health Organization (WHO) advised to all the counties to restrict the people's exit and the entry of the country[2, 20].

Now, almost every countries have been suffering seriously due to the corona infection. A large number of people is confirmed as the COVID-19 positive patients as well as a large number of people are in quarantine being asymptomatic. The exposed and the asymptomatic persons are more harmful one. The exposed classes are being infectious at any time as its incubation period is 2-14 days [21]. On the other hand the asymptomatic classes is most dangerous compared to any other classes, because the asymptomatic persons are not showing the symptoms of the disease as a result the people interacting with them 
are not taking any care as a consequence COVID-19 is spreading very rapidly [14].

Again, due to large number of infected cases and limited medical capacity in maximum countries the number of diagnosis tests for confirmation of COVID-19 infection is low. This fact also promotes the number of infected populations[22]. The harmfulness of COVID-19 is so high that upto $11^{\text {th }}$ May, 2020 nearly forty two lacks people have been infected among which 2,87,131 are died all over the world[21]. As the disease is spreading through interaction and no proper medicine is available till now, so maintaining social distancing and reducing interaction among the people is the only way to control the spreading of the disease. To maintain interaction among the people China Government adopted the lock-down policy and is able to control the spreading of the disease[22, 23]. Following China almost every countries have been now adopting this policy. Another measure to control this disease is to quarantine. Quarantine is the best measure to control the disease. If any COVID-19 positive case is identified then that person should be immediately hospitalized and his family members should be immediately quarantined along with their COVID-19 testing. Moreover all the persons who have come to the infected person should also be home quarantined for at least 14 days to avoid spreading of the disease.

Another way to spread the disease is by immigrants and also by migratory labours. In the Third World countries like India, Bangladesh and others there are many migratory labours who work in other countries or states. In the pandemic situation Government should allow them to come back their home place and then send them directly to quarantine centre. This can drastically reduce the outbreak size.

Moreover a large population come in those countries from other highly infected countries. So these countries have high chances of spreading this COVID-19 disease. To control and maintain the movements of the population, Government of India adopted lock-down policy for twenty one days in the first phase, which started from $25^{\text {th }}$ March, 2020 and it is now extended to $31^{\text {st }}$ May, 2020.

Our main goal of this work is to study the disease dynamics of COVID-19 by studying a deterministic compartmental model based on Indian scenario also to find some preventive measures for controlling its outbreak in India. Using the daily cases reported from India, we have estimated the model parameters, the effective reproduction number and made some predictions about the prevalence of the disease for India. 
Organization of the paper is as follows: In Section-2, we have studied the model formulation. Basic properties and the basic reproduction number of the proposed model have been studied in the Section-3 and Section 4, respectively. In Section 5, we studied the steady state analysis of disease free equilibrium point. Model fitting, parameter estimation, model validation and prediction have been done in Section 6. Sensitivity analysis has been done in Section 7. Computation of basic reproduction number from initial growth rate and effective reproduction number have been calculated in Section 8 . Some preventive measures have been proposed in Section 9. Finally, the concluding remarks are given in Section 10.

\section{Model Formulation}

In this work, we shall study the transmission mechanism of COVID-19 using a deterministic compartmental model. In order to formulate the model mathematically we have divided total population $N(t)$ into seven mutually exclusive compartments on the basis of their disease status. The Susceptible $(S(t))$, Exposed $(E(t))$, the class of people who are infected but not showing any symptom is known as Asymptomatic career. They are symptom less but has ability to transmit the virus. The class is denoted by $(A(t))$. There always exist a group of people who are infected and have some symptoms but not reported. So they are roaming about and not quarantined. This is the most vulnerable class is denoted by $(I(t))$. Again some infected persons having symptoms are reported. If their physical condition is not critical or they do not have any other disease like diabetes, hypertension nephritis they will be kept in home quarantined. This class is denoted as $(Q(t))$. The infected persons with severe symptoms are hospitalized. They belong to the class $(H(t))$. The recovered class $R(t)$ consists of those people who have been recovered from the COVID-19 disease getting testing report negative. Thus at any time $t$ total population $N(t)=S(t)+E(t)+A(t)+I(t)+Q(t)+H(t)+R(t)$. A susceptible person may be infected by the close contact with a infected person. In general, the quarantined and isolated persons are unable to transmit the virus to other susceptible persons but practically we observe that many staffs of quarantine and isolation centres such as doctors, nurses and other health staffs have been infected by such persons. Let the proportion $q_{1}$ of quarantined and $q_{2}$ of isolated individuals obey the rules of quarantine and isolation centres properly so that the proportions $\left(1-q_{1}\right)$ and $\left(1-q_{2}\right)$ of quarantined and isolated infected individuals, respectively, does not obey the rules of such centre properly and they are responsible to transmit the virus among the staffs of such centres. So, after getting infection due to the interaction with any individual of classes $A, I, Q, H$, an indi- 
vidual from $S$ class moves to the exposed class. Here we consider the rate of infection of the form $\lambda(S, E, A, I, Q, H, R)(1-d) S=\frac{\beta\left\{I+\rho A+\left(1-q_{1}\right) Q+\left(1-q_{2}\right) H\right\}}{N}(1-d) S$, where $\beta$ is the transmission rate of COVID-19 infection from symptomatic populations, $\rho$ is the ratio of the transmission rates of infection from asymptomatic and symptomatic infected individuals and the proportion $d$ of susceptible populations maintains safe distancing due to lock-down, personal awareness, and different awareness programmes. We have considered the constant recruitment rate $\pi$ in susceptible class and the natural death rate $\mu$. Exposed individuals move to three different compartments: asymptomatic, symptomatic and quarantined, symptomatic but not quarantined class separately at the rate $\sigma_{a}, \sigma_{q}, \sigma_{i}$, respectively. Symptomatic but not quarantined infected and symptomatic quarantined infected individuals have been detected and hospitalised at a rate $\eta_{i}, \eta_{q}$, respectively. Asymptomatic, symptomatic, quarantined, hospitalised individuals recover from the infection at a rate $\gamma_{a}, \gamma_{i}, \gamma_{q}$ and $\gamma_{h}$, respectively, and COVID-19 induced mortality rate is $\delta$. Under the above conditions the flow diagram of the COVID-19 transmission has been given in the Figure 1 and the transmission of the virus is governed by the following system of non-linear ODE:

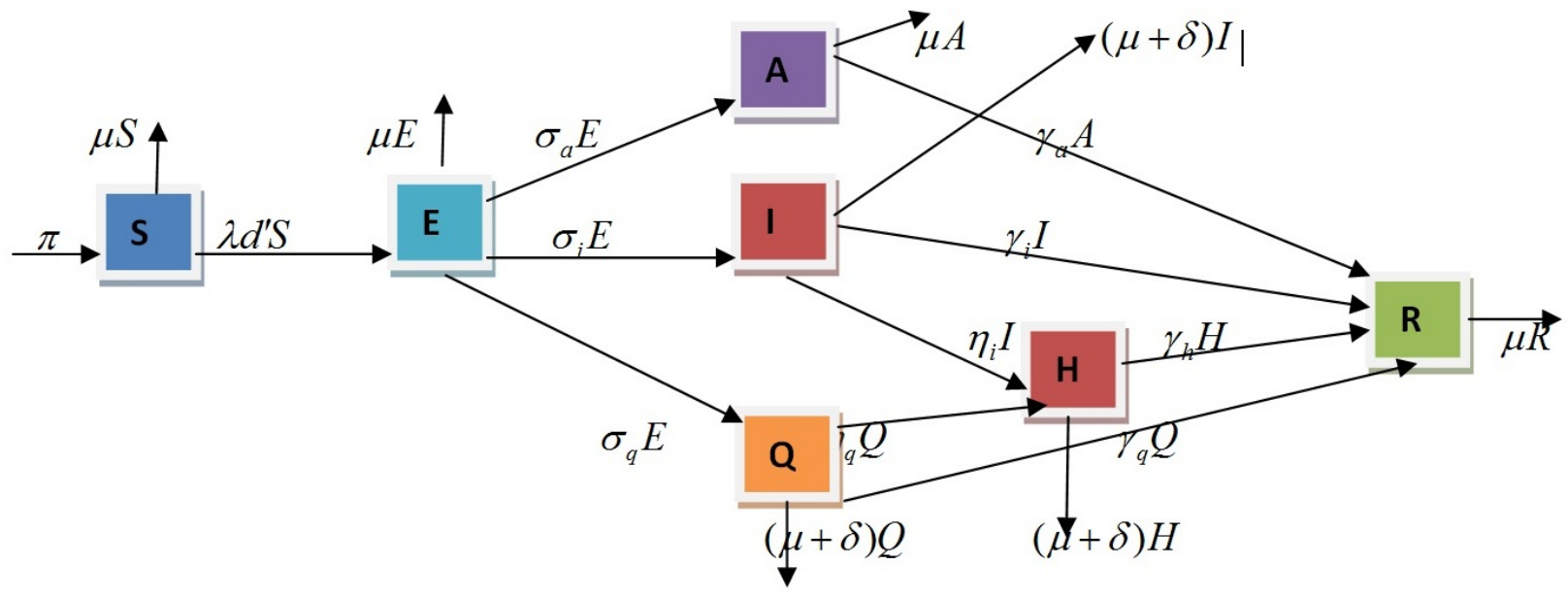

Figure 1: Flow diagram of disease transmission of COVID-19 


$$
\left\{\begin{array}{l}
\frac{d S}{d t}=\pi-\{\lambda(S, E, A, I, Q, H, R)(1-d)+\mu\} S \\
\frac{d E}{d t}=\lambda(S, E, A, I, Q, H, R)(1-d) S-\left(\sigma_{a}+\sigma_{i}+\sigma_{q}+\mu\right) E \\
\frac{d A}{d t}=\sigma_{a} E-\left(\gamma_{a}+\mu\right) A \\
\frac{d I}{d t}=\sigma_{i} E-\left(\eta_{i}+\gamma_{i}+\mu+\delta\right) I \\
\frac{d Q}{d t}=\sigma_{q} E-\left(\eta_{q}+\gamma_{q}+\mu+\delta\right) Q \\
\frac{d H}{d t}=\eta_{i} I+\eta_{q} Q-\left(\gamma_{h}+\mu+\delta\right) H \\
\frac{d R}{d t}=\gamma_{a} A+\gamma_{i} I+\gamma_{q} Q+\gamma_{h} H-\mu R,
\end{array}\right.
$$

with the initial conditions $S(0)>0, E(0) \geq 0, A(0) \geq 0, I(0)>0, Q(0) \geq 0, H(0) \geq 0, R(0) \geq 0$ and description of the parameters used in the model has been presented in Table 1.

Table 1: Interpretations of the model parameters

\begin{tabular}{ll}
\hline Parameters & Biological Meaning \\
\hline$d(0 \leq d \leq 1):$ & Proportion of susceptible population who obeys lock down strictly. \\
$q_{1}, q_{2}\left(0 \leq q_{1}, q_{2} \leq 1\right):$ & $\begin{array}{l}\text { Proportion of quarantine and isolation effect on effective contact rate, respectively. } \\
\text { Ratio of the transmission rates from asymptomatic and symptomatic class. }\end{array}$ \\
$\beta:$ & Virus transmission rate from symptomatic infected to susceptible population. \\
$\sigma_{a}, \sigma_{i}, \sigma_{q}:$ & Rates of conversion from exposed to $A, I$ and $Q$ classes, respectively. \\
$\eta_{i}, \eta_{q}:$ & Rates of hospitalisation from $I$ and $Q$ classes, respectively. \\
$\gamma_{a}, \gamma_{i}, \gamma_{q}, \gamma_{h}:$ & Recovery rates from $A, I, Q, H$ classes, respectively. \\
$\pi:$ & Recruitment rate of human. \\
$\mu, \delta:$ & Normal and disease induced death rate of human, respectively. \\
\hline
\end{tabular}

\section{Positivity and Boundedness of Solutions}

In this section, we shall study the basic properties of the COVID-19 model (1). The model will be biologically meaningful if all the variables are non-negative for $t \geq 0$ in other wards solution with nonnegative initial conditions will remain non-negative for all time, which we shall study in the following 
lemma.

Lemma: 1. Let us suppose $F(t)=(S, E, A, I, Q, H, R)$ along with the initial conditions $F(0) \geq 0$ then the solutions $F(t)$ of the COVID-19 model (1) are non-negative for all $t \geq 0$.

Proof. Multiplying the first equation of (1) by its integrating factor $\exp \left\{\mu t+\int_{0}^{t} \lambda(\tau) d \tau\right\}$ and arranging the equation, we get

$$
\begin{gathered}
\frac{d}{d t}\left[S(t) \exp \left\{\mu t+\int_{0}^{t} \lambda(\tau) d \tau\right\}\right]=\pi \exp \left\{\mu t+\int_{0}^{t} \lambda(\tau) d \tau\right\} \\
\text { or, } S(t) \exp \left\{\mu t+\int_{0}^{t} \lambda(\tau) d \tau\right\}=S(0)+\pi \int_{0}^{t} \exp \left\{\mu y+\int_{0}^{y} \lambda(\tau) d \tau\right\} d y \\
\text { or, } S(t)=\left\{S(0)+\pi \int_{0}^{t} \exp \left\{\mu y+\int_{0}^{y} \lambda(\tau) d \tau\right\} d y\right\}\left\{\exp \left\{-\mu t-\int_{0}^{t} \lambda(\tau) d \tau\right\}\right\} \geq 0 .
\end{gathered}
$$

Similarly, it can be established that $E(t) \geq 0, A(t) \geq 0, I(t) \geq 0, Q(t) \geq 0, H(t) \geq 0, R(t) \geq 0$.

Thus, $F(t) \geq 0$ for all $t>0$.

The dynamical nature of the COVID-19 model (1) shall be studied in the feasible closed region:

$$
\Omega=\left\{(S, E, A, I, Q, H, R) \in \mathbb{R}_{+}^{7}: S+E+A+I+Q+H+R \leq \frac{\pi}{\mu}\right\} .
$$

It will be established that the closed region $\Omega$ is a positively invariant and an attractor of all positive solutions of the COVID-19 model (1).

Lemma: 2. The closed region $\Omega$ is a positively invariant set for the COVID-19 model (1) with the initial conditions in $\mathbb{R}_{+}^{7}$.

Proof. Adding all the seven component equations of the model (1) and using the relation $N=S+E+$ $A+I+Q+H+R$, we have

$$
\frac{d N}{d t}=\pi-\delta(I+Q+H)-\mu N
$$

which implies

$$
\frac{d N}{d t} \leq \pi-\mu N
$$

or,

$$
\frac{d N}{d t}+\mu N \leq \pi
$$


Multiplying both sides by the integrating factor, integrating, and using the initial conditions, we get

$$
N(t) \leq N(0) e^{-\mu t}+\frac{\pi}{\mu}\left(1-e^{-\mu t}\right)
$$

It is clear from (3) that $N(t) \leq \frac{\pi}{\mu}$ if $N(0) \leq \frac{\pi}{\mu}$. Thus, $\Omega$ is a positively invariant set under the flow presented in the COVID-19 model (1). Further, if $N(0)>\frac{\pi}{\mu}$ then $N(t)$ approaches to $\frac{\pi}{\mu}$ and the number of infected population in the classes $E, A, I, Q$ and $H$ approach to zero for large $t$. So, all solutions in $\mathbb{R}_{+}^{7}$ of the model (1) eventually enter in $\Omega$ i.e. it is an attracting set.

Thus, the COVID-19 model (1) is well posed biologically and mathematically in the invariant set $\Omega$ [24].

\section{Basic Reproduction Number}

The basic reproduction number plays important role in controlling and spreading the disease. It is defined as the number of secondary infections i.e. the number of new infections spread by a single infected person. Analytically it can be found easily if the disease free equilibrium of the system exists. Diekmann et. al. [25] and Van den Driessche and Watmough [26] proposed a generalized approach to determine the basic reproduction number which is known as the next generation matrix approach. For the system (1) the disease free equilibrium point (DFE) is $E_{0}\left(\frac{\pi}{\mu}, 0,0,0,0,0,0\right)$ and hence basic reproduction number of the proposed problem exists.

Here, we decompose the right hand side of the system (1) corresponding to the infected compartments $E, A, I, Q$ and $H$ as $\mathscr{F}-\mathscr{V}$,

where $\mathscr{F}=\left(\begin{array}{c}\frac{\beta\left(\rho A+I+q_{1} Q+q_{2} H\right)(1-d) S}{N} \\ 0 \\ 0 \\ 0 \\ 0\end{array}\right)$ and $\mathscr{V}=\left(\begin{array}{c}k_{1} E \\ -\left(\sigma_{a} E-k_{2} A\right) \\ -\left(-k_{3} I+\sigma_{i} E\right) \\ -\left(-k_{4} Q+\sigma_{q} E\right) \\ -\left(-k_{5} H+\eta_{i} I+\eta_{q} Q\right)\end{array}\right)$.

Here $k_{1}=\sigma_{a}+\sigma_{i}+\sigma_{q}+\mu, k_{2}=\gamma_{a}+\mu, k_{3}=\eta_{i}+\gamma_{i}+\mu+\delta, k_{4}=\eta_{q}+\gamma_{q}+\delta+\mu, k_{5}=\gamma_{h}+\delta+\mu$. 
Let us define

$$
F=\frac{\partial \mathscr{F}}{\partial x_{j}}\left(E_{0}\right)=\left(\begin{array}{ccccc}
0 & \beta \rho(1-d) & \beta(1-d) & \beta\left(1-q_{1}\right)(1-d) & \beta\left(1-q_{2}\right)(1-d) \\
0 & 0 & 0 & 0 & 0 \\
0 & 0 & 0 & 0 & 0 \\
0 & 0 & 0 & 0 & 0 \\
0 & 0 & 0 & 0 & 0
\end{array}\right)
$$

and

$$
V=\frac{\partial \mathscr{V}}{\partial x_{j}}\left(E_{0}\right)=\left(\begin{array}{ccccc}
-k_{1} & \beta q_{1}(1-d) & \beta(1-d) & \beta \rho(1-d) & \beta_{2}(1-d) \\
\sigma_{a} & 0 & 0 & -a & 0 \\
\sigma_{i} & 0 & -k_{3} & 0 & 0 \\
\sigma_{q} & -k_{4} & 0 & 0 & 0 \\
0 & \eta_{q} & \eta_{i} & 0 & -k_{5}
\end{array}\right)
$$

for $x_{j}=E, A, I, Q, H$. Since the basic reproduction number is the spectral radius of the next generation matrix $F V^{-1}$, hence we have the basic reproduction number for the considered model is

$$
\begin{gathered}
R_{0}=\frac{\beta \rho(1-d) \sigma_{a}}{k_{1} k_{2}}+\frac{\beta(1-d) \sigma_{i}}{k_{1} k_{3}}+\frac{\beta\left(1-q_{1}\right) \sigma_{q}(1-d)}{k_{1} k_{4}}+\frac{\beta\left(1-q_{2}\right)\left(\eta_{i} k_{4} \sigma_{i}+\eta_{q} k_{3} \sigma_{q}\right)(1-d)}{k_{1} k_{3} k_{4} k_{5}} \\
=R_{0 A}+R_{0 I}+R_{0 Q}+R_{0 H},
\end{gathered}
$$

where $R_{0 A}, R_{0 I}, R_{0 Q}$ and $R_{0 H}$ are the parts of basic reproduction number contributed by asymptomatic infected class, symptomatic but non-quarantined infected class, symptomatic and quarantined infected class and hospitalised infected class, respectively.

\section{Steady State Analysis of DFE}

The model has the DFE $E_{0}\left(\frac{\pi}{\mu}, 0,0,0,0,0,0\right)$, whose stability analysis has been done through the following theorem.

Theorem 1. If $R_{0}>1$ then the DFE $E_{0}$ is unstable and it is stable if $R_{0}<1$.

Proof. : The variational matrix corresponding to the system (1) at DFE $E_{0}(\pi / \mu, 0,0,0,0,0,0)$ is 


$$
J\left(E_{0}\right)=\left(\begin{array}{ccccccc}
-\mu & 0 & -\beta \rho(1-d) & -\beta(1-d) & -\beta\left(1-q_{1}\right)(1-d) & -\beta\left(1-q_{2}\right)(1-d) & 0 \\
0 & -k_{1} & \beta \rho(1-d) & \beta(1-d) & \beta\left(1-q_{1}\right)(1-d) & \beta\left(1-q_{2}\right)(1-d) & 0 \\
0 & \sigma_{a} & -k_{2} & 0 & 0 & 0 & 0 \\
0 & \sigma_{i} & 0 & -k_{3} & 0 & 0 & 0 \\
0 & \sigma_{q} & 0 & 0 & -k_{4} & 0 & 0 \\
0 & 0 & 0 & \eta_{i} & \eta_{q} & -k_{5} & 0 \\
0 & 0 & \gamma_{a} & \gamma_{i} & \gamma_{q} & \gamma_{h} & -\mu
\end{array}\right) .
$$

The eigenvalues of the variational matrix $J\left(E_{0}\right)$ are $-\mu,-\mu, \lambda_{1}, \lambda_{2}, \lambda_{3}, \lambda_{4}, \lambda_{5}$, where $\lambda_{i}(i=1,2,3,4,5)$ are the roots of the following equation:

$$
P(\lambda) \equiv \frac{\sigma_{a} \beta \rho(1-d)}{\left(k_{1}+\lambda\right)\left(k_{2}+\lambda\right)}+\frac{\sigma_{i} \beta(1-d)}{\left(k_{1}+\lambda\right)\left(k_{3}+\lambda\right)}+\frac{\sigma_{q} \beta(1-d)\left(1-q_{1}\right)}{\left(k_{1}+\lambda\right)\left(k_{4}+\lambda\right)}+\frac{\eta_{q} \sigma_{q} \beta(1-d)\left(1-q_{2}\right)}{\left(k_{1}+\lambda\right)\left(k_{4}+\lambda\right)\left(k_{5}+\lambda\right)}+\frac{\eta_{i} \sigma_{i} \beta(1-d)\left(1-q_{2}\right)}{\left(k_{1}+\lambda\right)\left(k_{3}+\lambda\right)\left(k_{5}+\lambda\right)}-1=0 .
$$

Then $P(0)=R_{0}-1$. There are two cases.

Case I: Suppose $R_{0}>1$. Then $P(0)>0$. Again, $P(\lambda)$ tends to -1 as $\lambda$ tends to $\infty$. Since $P(\lambda)$ is a continuous function of $\lambda$, hence the Bolzano theorem on continuous function implies that $P\left(\lambda_{i}\right)=0$ for some $\lambda_{i}>0$. Thus, at least one eigenvalue of the variational matrix must be positive. Therefore, in this case the DFE $E_{0}$ is unstable.

Case II: Suppose $R_{0}<1$. Then $P(0)<0$.

If possible let us assume that $P(\lambda)=0$ has a root of the form $x+i y$, where $x, y \in \mathbb{R}$ and $x \geq 0$. Then $P(x+i y)=0$.

Again, $|P(x+i y)+1| \leq \frac{\sigma_{a} \beta \rho(1-d)}{\left|k_{1}+\lambda\right|\left|k_{2}+\lambda\right|}+\frac{\sigma_{i} \beta(1-d)}{\left|k_{1}+\lambda\right|\left|k_{3}+\lambda\right|}+\frac{\sigma_{q} \beta(1-d)\left(1-q_{1}\right)}{\left|k_{1}+\lambda\right|\left|k_{4}+\lambda\right|}+\frac{\eta_{q} \sigma_{q} \beta(1-d)\left(1-q_{2}\right)}{\left|k_{1}+\lambda\right|\left|k_{4}+\lambda\right|\left|k_{5}+\lambda\right|}+\frac{\eta_{i} \sigma_{i} \beta(1-d)\left(1-q_{2}\right)}{\left|k_{1}+\lambda\right|\left|k_{3}+\lambda\right|\left|k_{5}+\lambda\right|}$ $\leq \frac{\sigma_{a} \beta \rho(1-d)}{\left(k_{1}+x\right)\left(k_{2}+x\right)}+\frac{\sigma_{i} \beta(1-d)}{\left(k_{1}+x\right)\left(k_{3}+x\right)}+\frac{\sigma_{q} \beta(1-d)\left(1-q_{1}\right)}{\left(k_{1}+x\right)\left(k_{4}+x\right)}+\frac{\eta_{q} \sigma_{q} \beta(1-d)\left(1-q_{2}\right)}{\left(k_{1}+x\right)\left(k_{4}+x\right)\left(k_{5}+x\right)}+\frac{\eta_{i} \sigma_{i} \beta(1-d)\left(1-q_{2}\right)}{\left(k_{1}+x\right)\left(k_{3}+x\right)\left(k_{5}+x\right)}$ $\leq \frac{\sigma_{a} \beta \rho(1-d)}{k_{1} k_{2}}+\frac{\sigma_{i} \beta(1-d)}{k_{1} k_{3}}+\frac{\sigma_{q} \beta(1-d)\left(1-q_{1}\right)}{k_{1} k_{4}}+\frac{\eta_{q} \sigma_{q} \beta(1-d)\left(1-q_{2}\right)}{k_{1} k_{4} k_{5}}+\frac{\eta_{i} \sigma_{i} \beta(1-d)\left(1-q_{2}\right)}{k_{1} k_{3} k_{5}}=R_{0}<1$, which implies that $1<1$. This is clearly a contradiction. Hence, all the roots of the equation $P(\lambda)=0$ have the form $x+i y$, where $x, y \in \mathbb{R}$ and $x<0$. Thus, in this case the DFE is stable.

Hence the theorem is proved.

\section{Model Fitting-Parameter Estimation and Model Validation}

In this section, we shall estimate the model parameters by using the reported data of COVID-19. It has been spread all over the world at a high alarming rate. So, the control of the transmission of this virus as early as possible is very essential for the existence of human civilization. On the other hand, the natural 
history and dynamics of the novel coronavirus is unknown till date. In this context parameter estimation is an important task to study its dynamics.

\subsection{Model Fitting and Parameter Estimation}

We have estimated the key model parameters connected to COVID-19 by fitting our proposed model to the reported cases of the ongoing COVID-19 pandemic in India. Although this data set is incomplete as the pandemic is ongoing but we shall use it for controlling this pandemic after studying its early dynamics. Actually the COVID-19 outbreak starts in India from $1^{\text {st }}$ March 2020 as from that date the new infected cases are reported continuously. On the other hand, a major preventive measure was taken by the Government of India by implementing a countrywide lock-down from $25^{\text {th }}$ March 2020 that has been continuing upto $17^{\text {th }}$ May 2020 and may continue further if necessary. So we consider two sets of data: first one (set-1) is the collection of the cases reported during the period $1^{\text {st }}$ March to $24^{\text {th }}$ April of 2020. But before the implementation of lock-down (25 ${ }^{\text {th }}$ March, 2020) many exposed, symptomatic as well as asymptomatic infected persons came in India from other COVID-19 pandemic countries who were neither detected nor reported properly. So there remains uncertainty among the data reported during the period $1^{\text {st }}$ March to $24^{\text {th }}$ March, 2020. Thus we consider the second set (set-2) of data reported during the period $25^{\text {th }}$ March to $24^{\text {th }}$ April, 2020 by avoiding the problem in above said period. We have fitted the model to both the sets of data. The fitting to the first set has been presented in Figure 2(a)-(b) and the estimated parameters have been summarised in Table 2 whereas the fitting of the second set has been illustrated in Figure 3(a)-(b) and the corresponding parameters have been put in Table 3. The results show that the second one is best fit and we shall use this set for model prediction.

We have fitted the model to cumulative cases in India, which are obtained from [21]. Our model predicted cumulative new infected cases $(q(t))$ satisfy the following equation:

$$
q(t, \Phi)=q(0)+\int_{0}^{t}\left(\eta_{i} I(\tau)+\eta_{q} Q(\tau)\right) d \tau
$$

We solve the model equations numerically and use the solutions to determine the best-fit model parameters by using a non-linear least squares regression technique which minimizes the sum of the squared residuals

$$
R(\Phi)=\sum_{J=1}^{n}\left(q_{t_{j}}(\Phi)-\bar{q}_{t_{j}}\right)^{2}
$$


where $\Phi=\left(\beta, \sigma_{a}, \sigma_{i}, \sigma_{q}, \gamma_{a}, \gamma_{i}, \gamma_{q}, \gamma_{h}, \eta_{i}, \eta_{q}, \delta, \rho, d\right)$ is a set of model parameters to be estimated. $q_{t_{j}}(\Phi)$ and $\bar{q}_{t_{j}}$ are cumulative number of infected population accordingly by model prediction and by reported data, respectively. Here $n$ denotes the total number of data points available for the fitting process.

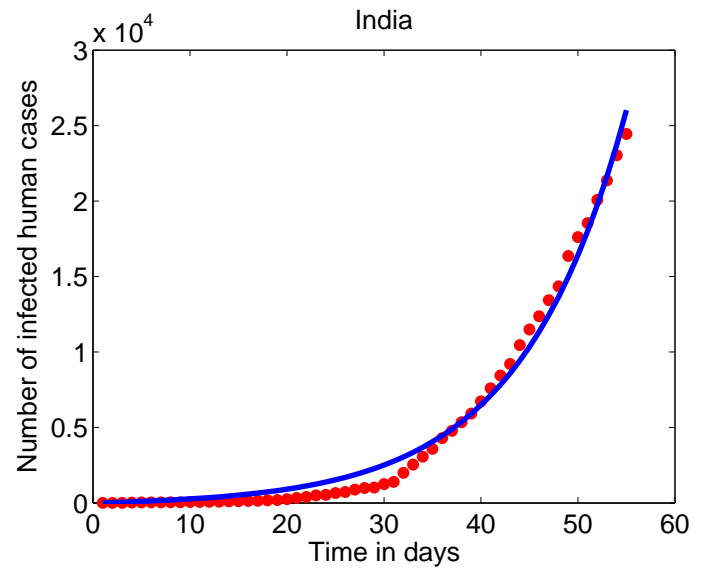

(a)

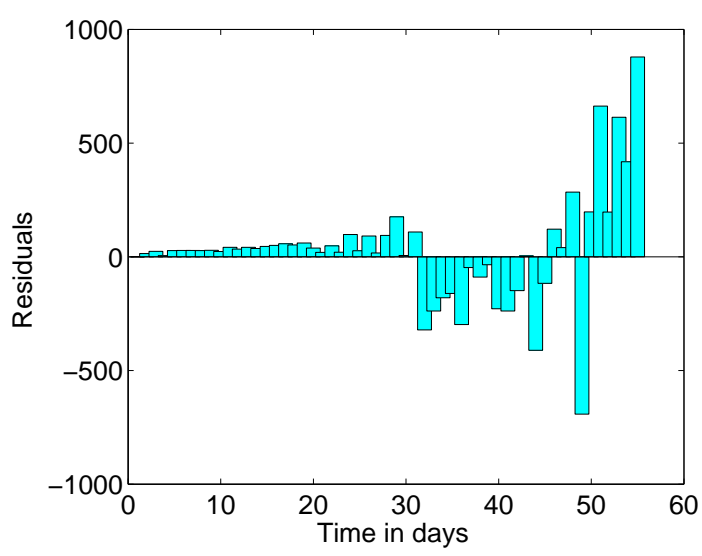

(b)

Figure 2: (a) Model simulation to the cumulative reported cases from $1^{\text {st }}$ March to $24^{\text {th }}$ April, 2020 in India, the red dots denote the reported infected cases and blue line presents the model predicted infected cases, (b) Residuals of the corresponding data fitting.

In order to fit the model with the reported cases in India, we have the total population in India as 1352642280 [35] considered as susceptible for COVID-19 that is the initial number of susceptible $S(0)=$ 1352642280. The birth rate is $18.2 / 1000$ per year [35]. So the daily recruitment rate in India is 67446.82 . The average life span of India is 69.7 [35] years and hence the death rate is 0.000039074 .

For the first data set, initially that is on the $1^{\text {st }}$ March, 2020 the reported cumulative infected cases was 3 , which is the number of cases that has been considered as initial hospitalised cases and initial cumulative cases that is $H(0)=3$ and $q(0)=3$. There are no available information about the initial number of exposed, asymptomatic infected and symptomatic but non-quarantined infected (that is not reported), so we have to estimate $E(0), A(0), I(0)$ for the both data sets and we assume that $q_{1}=0.94, q_{2}=0.90$. Using the above initial conditions, recruitment rate and normal death rate, we have fitted our proposed model (Figure 2(a)) to the reported data [21] and corresponding residuals are presented in Figure 2(b). We have estimated all other key parameters including the basic reproduction number. According to our estimation we have $E(0)=111, A(0)=16, I(0)=10$ and 
Table 2: List of the model parameters and their sensitivity indices for COVID-19 pandemic in India, estimated from the data from $1^{\text {st }}$ March to $24^{\text {th }}$ April, 2020

\begin{tabular}{|c|c|c|c|}
\hline Parameters & Values & Sources & Sensitivity Indices \\
\hline$\pi$ & $67446.82054 \mathrm{day}^{-1}$ & {$[21]$} & - \\
\hline$\mu$ & 0.0000391 day $^{-1}$ & {$[21]$} & -0.00076 \\
\hline$\beta$ & 1.11525 day $^{-1}$ & Estimated & 1.000000000 \\
\hline$\sigma_{a}$ & 0.08275 day $^{-1}$ & Estimated & 0.44301 \\
\hline$\sigma_{i}$ & 0.35872 day $^{-1}$ & Estimated & -0.08026 \\
\hline$\sigma_{q}$ & 0.33511 day $^{-1}$ & Estimated & -0.36270 \\
\hline$\gamma_{a}$ & 0.03435 day $^{-1}$ & Estimated & -0.54894 \\
\hline$\gamma_{i}$ & 0.01496 day $^{-1}$ & Estimated & -0.01794 \\
\hline$\gamma_{q}$ & $0.05481 \mathrm{day}^{-1}$ & Estimated & -0.00619 \\
\hline$\gamma_{h}$ & $0.09310 \mathrm{day}^{-1}$ & Estimated & -0.08415 \\
\hline$\eta_{i}$ & $0.26190 \mathrm{day}^{-1}$ & Estimated & -0.25181 \\
\hline$\eta_{q}$ & 0.51323 day $^{-1}$ & Estimated & 0.00152 \\
\hline$\delta$ & 0.04142 day $^{-1}$ & Estimated & -0.09177 \\
\hline$q_{1}$ & 0.94 & Assumed & -0.14651 \\
\hline$q_{2}$ & 0.90 & Assumed & -1.09464 \\
\hline$\rho$ & 0.80576 & Estimated & 0.54957 \\
\hline$d$ & 0.52674 & Estimated & -1.11299 \\
\hline$R_{0}$ & 2.39745 & Estimated & 1.00000 \\
\hline
\end{tabular}


the value of the basic reproduction is $R_{0}=2.397448679$ among which the contribution of the asymptomatic infected class is $R_{0 A}=1.317554127$. Similarly the contribution of the symptomatic but nonquarantined infected class, symptomatic and quarantined infected class and hospitalised infected class are $R_{0 I}=0.7658798290, R_{0 Q}=0.02241962187, R_{0 H}=0.2915951005$, respectively. As the contribution of the asymptomatic infected class $\left(R_{0 A}\right)$ is largest compared to the others, so we have to focus on the reduction of $R_{0 A}$ through some preventive strategies, which will be discussed in detail in later section.

For the second data set, initially i.e. on $25^{\text {th }}$ March, 2020 the cumulative number of infected population $q(0)=657, H(0)=657, S(0)=1352642280$ and we assume $Q(0)=647$. The other initial conditions i.e $E(0), A(0), I(0)$ are estimated. Under the above initial conditions the model fitting with the second set of data has been presented in Figure 3(a) and corresponding residuals have been presented in Figure 3(b). According to our estimation, we have $E(0)=1131, A(0)=506, I(0)=482$ and the basic reproduction is $R_{0}=2.414190966$ among which the contributions of different infected classes are $R_{0 A}=1.842473415, R_{0 I}=0.3910030945, R_{0 Q}=0.01860012179, R_{0 H}=0.1621143316$.

In both Figure 2(a)-(b) and Figure 3(a)-(b), we have illustrated the cumulative number of real cases and the model predicted cases with corresponding residuals for the set- 1 and set-2, respectively. The randomness of the residuals for both cases suggests that the fitness are good for each cases.

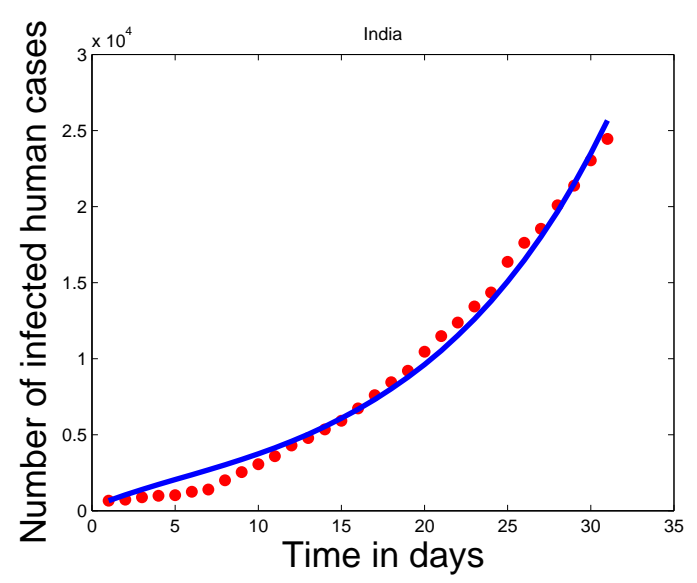

(a)

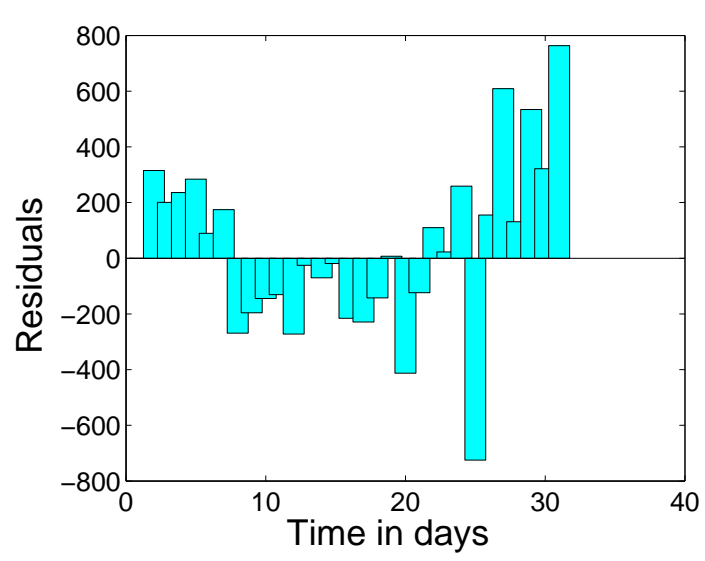

(b)

Figure 3: (a) Model simulation to the cumulative reported cases from $25^{\text {th }}$ March to $24^{\text {th }}$ April, 2020 in India, the red dots denote the reported infected cases and blue line presents the model predicted infected cases, (b) Residuals of the corresponding data fitting. 
Table 3: List of the model parameters and their sensitivity indices for COVID-19 pandemic in India estimated from the data from $25^{\text {th }}$ March to $24^{\text {th }}$ April, 2020

\begin{tabular}{|c|c|c|c|}
\hline Parameters & Values & Sources & Sensitivity Indices \\
\hline$\pi$ & 67446.82054 day $^{-1}$ & {$[21]$} & - \\
\hline$\mu$ & $0.0000391 \mathrm{day}^{-1}$ & {$[21]$} & -0.00065 \\
\hline$\beta$ & 0.88689 day $^{-1}$ & Estimated & 1.0000 \\
\hline$\sigma_{a}$ & 0.24176 day $^{-1}$ & Estimated & 0.44295 \\
\hline$\sigma_{i}$ & 0.24757 day $^{-1}$ & Estimated & -0.13547 \\
\hline$\sigma_{q}$ & 0.26556 day $^{-1}$ & Estimated & -0.30743 \\
\hline$\gamma_{a}$ & $0.05311 \mathrm{day}^{-1}$ & Estimated & -0.76262 \\
\hline$\gamma_{i}$ & $0.05090 \mathrm{day}^{-1}$ & Estimated & -0.02561 \\
\hline$\gamma_{q}$ & $0.05071 \mathrm{day}^{-1}$ & Estimated & -0.00435 \\
\hline$\gamma_{h}$ & 0.07048 day $^{-1}$ & Estimated & -0.03394 \\
\hline$\eta_{i}$ & $0.26267 \mathrm{day}^{-1}$ & Estimated & -0.10165 \\
\hline$\eta_{q}$ & 0.39787 day $^{-1}$ & Estimated & 0.00255 \\
\hline$\delta$ & $0.06891 \mathrm{day}^{-1}$ & Estimated & -0.07377 \\
\hline$q_{1}$ & 0.94 & Assumed & -0.12070 \\
\hline$q_{2}$ & 0.90 & Assumed & -0.60436 \\
\hline$\rho$ & 0.67047 & Estimated & 0.76318 \\
\hline$d$ & 0.48576 & Estimated & -0.94461 \\
\hline$R_{0}$ & 2.41419 & Estimated & 1.00000 \\
\hline
\end{tabular}

\subsection{Model Validation and Prediction}

In this subsection, we validate the model by comparing the model predictions with the reported data which was not used for fitting process using both sets of parameters. We have compared the model predicted daily cases with the reported daily cases with the help of a bar diagram in India from $25^{\text {th }}$ April to $10^{\text {th }}$ May, 2020. The model predicted cases estimated from the first set of parameters have been given in Figure 4(a) whereas the predictions estimated from the second set have been given in Figure 4(b). 
Here the blue colour bar denotes predicted daily new cases and red colour bar denotes reported cases. From the Figure 4(a) and (b), it is clear that the second one gives more perfect estimation compared to the first one.

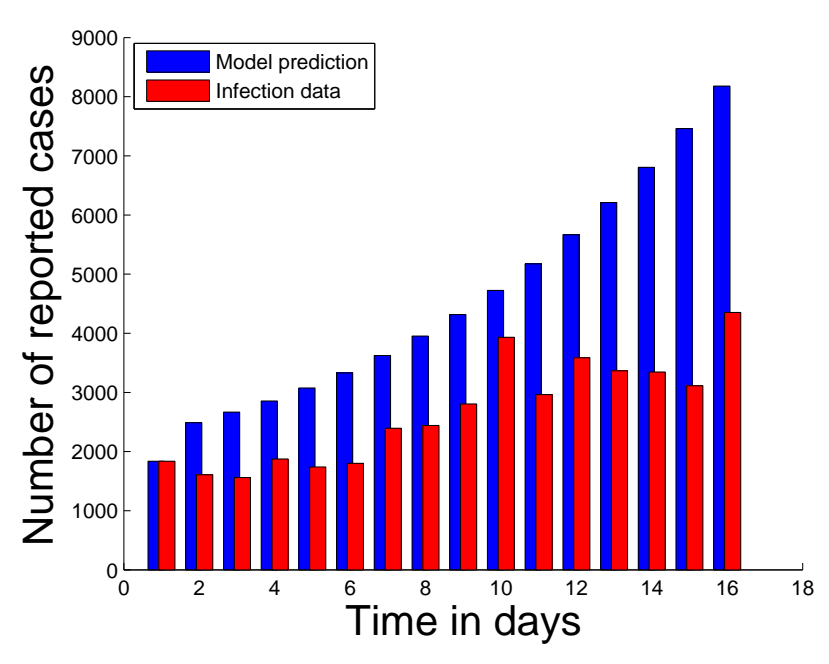

(a)

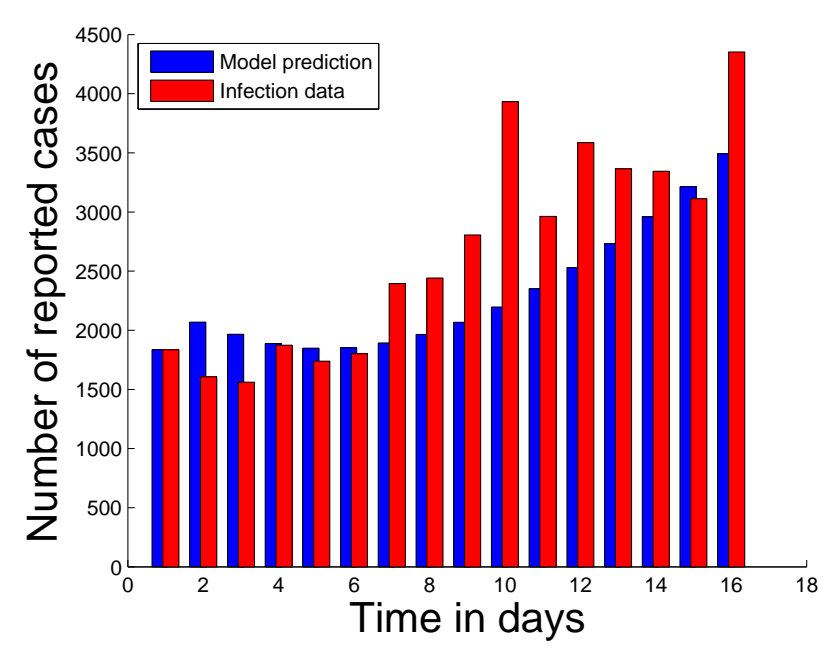

(b)

Figure 4: Bar diagram of the daily infected cases where red bar denotes the reported case and blue bar denotes the model predicted case from (a) set-1 parametric values, (b) set-2 parametric values, for $25^{\text {th }}$ April to $10^{\text {th }}$ May, 2020.

The best estimator also can be justified from the standard error of the two cases. The standard error corresponding to the model fitting to the first set of parameters is $2.8264954 \times 10^{7}$ and the error for the fitting to the second set is $1.630582 \times 10^{7}$. So, the second one is better fitting compared to the first. In reality, many exposed, asymptomatic infected, symptomatic infected persons came in India before $25^{\text {th }}$ March, 2020 (before the lock-down) from other COVID-19 affected countries, who were not detected properly. So, there is uncertainty in reported data before $25^{\text {th }}$ March, 2020. So, we are not considering the data which reported before on that date. From the Figure 4(a) and 4(b), it is clear that the prediction based on second set of parameters is more perfect compared to the first one. For the above said reasons, we shall choose the second set of parametric values which are estimated from the real data reported from $25^{\text {th }}$ March to $24^{\text {th }}$ April, 2020 to study the future trend of the outbreak and for model prediction.

In order to study the future trend of outbreak and predict from the model, we find the time series for the cumulative infected number of population in Figure 5(a) and for different infected classes in Figure 5(b) using the same initial conditions used in data fitting process and estimated parametric values for one 
year that is for 365 days from $25^{\text {th }}$ March, 2020. From the Figure 5(a), it is clear that at that time the total number of infected population will be $6.345 \times 10^{8}$. The Figure $5(\mathrm{~b})$ indicates that the number of symptomatic infected population will be maximum on around $146^{\text {th }}$ day starting from $25^{\text {th }}$ March, 2020 i.e on around $17^{\text {th }}$ August, 2020, the hospitalised case will be maximum on around $23^{\text {th }}$ August, 2020 and the asymptomatic case will be maximum on $27^{\text {th }}$ August, 2020. The model also predicts that the disease will be fully controlled after 365 days.

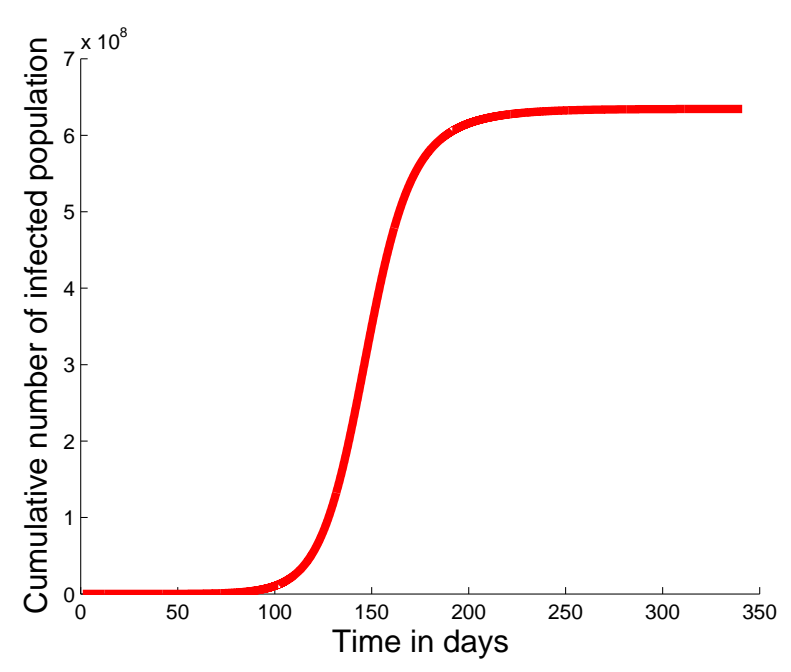

(a)

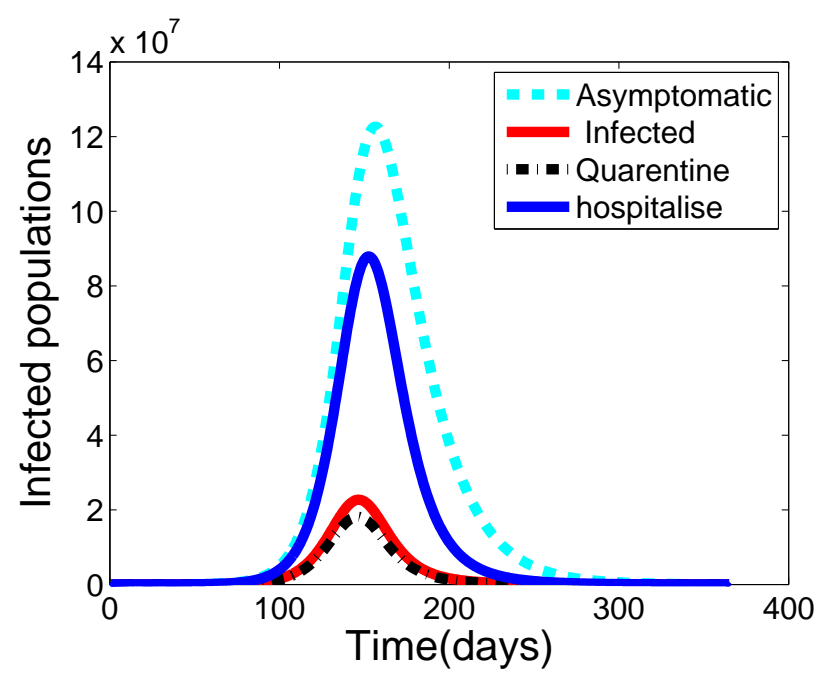

(b)

Figure 5: Time series for (a) cumulative number of infected population and (b) different infected population using the estimated parametric values and same initial conditions.

\section{Sensitivity Analysis}

COVID-19 has been spreading globally at a high alarming rate and it is a threat for human civilization. So prevention and control of this viral disease is very important task at this moment. In this context first of all we have to identify and quantify the influential model parameters. In order to determine such parameters we shall evaluate sensitivity index of the basic reproduction number with respect to different parameters. Using the normalized forward sensitivity method [27, 28], we obtained sensitivity index $S_{\alpha}=\frac{\partial R_{0}}{\partial \alpha} \frac{\alpha}{R_{0}}$, where $\alpha$ is the characteristic parameter whose sensitivity on $R_{0}$ has to be determined. The calculated sensitivity indices with respect to each of the model parameters using both sets of parametric values have been presented in the last column of Table 2 and Table 3, respectively. The significance of this index is 
that the index with higher in magnitude implies high sensitivity of that parameter on $R_{0}$. The significance of the positive (or negative) sign of sensitivity index is that $R_{0}$ increases (or decreases) as the parameter $\alpha$ increases. Our findings show that the most influential parameters are lock-down factor $(d)$, virus transmission rate $(\beta)$, ratio of the virus transmission rates from asymptomatic and symptomatic infected population $(\rho)$, recovery rate from asymptomatic infected class $\left(\gamma_{a}\right)$, disease induced death rate $(\delta)$, transmission rate from exposed to asymptomatic infected class $\left(\sigma_{a}\right)$ and recovery rate from hospitalised infected class $\left(\gamma_{h}\right)$. Such indices can guide to identify and quantify the effective control and preventive strategies.

\section{Basic Reproduction Number of the COVID-19 Outbreak in India}

In this section, we shall estimate the basic reproduction number from the actual data and study the effective basic reproduction number for the outbreak in India.

\subsection{Estimation of $R_{0}$ from Actual Data of COVID-19 Outbreak in India}

There are several mathematical as well as statistical techniques to compute the basic reproduction number $\left(R_{0}\right)$ for infectious diseases from the actual pandemic data [30]. In this section, we estimate the basic reproduction number $R_{0}$ from initial growth phase of the COVID-19 epidemic in India [31]. We assume that at the early stage of pandemic the cumulative number of cases $q(t)$ varies as $\exp (\Lambda t)$ [29] i.e. $q(t) \propto \exp (\Lambda t)$, where $\Lambda$ is the force of infection. Similarly the number of exposed, asymptomatic, symptomatic but non-quarantined, symptomatic and quarantined, hospitalised infected population vary with $\exp (\Lambda t)$. So, we have

$$
\left\{\begin{array}{l}
E \sim E_{0} \exp (\Lambda t) \\
A \sim A_{0} \exp (\Lambda t) \\
I \sim I_{0} \exp (\Lambda t) \\
Q \sim Q_{0} \exp (\Lambda t) \\
H \sim H_{0} \exp (\Lambda t),
\end{array}\right.
$$

where $E_{0}, A_{0}, I_{0}, Q_{0}$ and $H_{0}$ are constants. Again, we assume that the number of non-susceptible population for COVID-19 in India be negligible i.e. $S(t)=\pi / \mu$. Now substituting (6) into the model equation 
(1), we have

$$
\left\{\begin{array}{l}
\left(\Lambda+k_{1}\right) E_{0}=\beta(1-d)\left\{I_{0}+\rho A_{0}+\left(1-q_{1}\right) Q_{0}+\left(1-q_{2}\right) H_{0}\right\} \\
\left(\Lambda+k_{2}\right) A_{0}=\sigma_{a} E_{0} \\
\left(\Lambda+k_{3}\right) I_{0}=\sigma_{i} E_{0} \\
\left(\Lambda+k_{4}\right) Q_{0}=\sigma_{q} E_{0} \\
\left(\Lambda+k_{5}\right) H_{0}=\eta_{i} I_{0}+\eta_{q} Q_{0} .
\end{array}\right.
$$

Using the equation (7) in the expression $R_{0}$ given in (4), we have the following relation between the basic reproduction number $\left(R_{0}\right)$ and the force of infection $(\Lambda)$ :

$$
R_{0}=\frac{\Lambda+k_{1}}{k_{1}} \frac{\frac{\sigma_{i}}{k_{3}}+\frac{\rho \sigma_{a}}{k_{2}}+\frac{\left(1-q_{1}\right) \sigma_{q}}{k_{4}}+\frac{\left(1-q_{2}\right)\left(\eta_{i} k_{4} \sigma_{i}+\eta_{q} k_{3} \sigma_{q}\right)}{k_{3} k_{4} k_{5}}}{\frac{\sigma_{i}}{\Lambda+k_{3}}+\frac{\rho \sigma_{a}}{\Lambda+k_{2}}+\frac{\left(1-q_{1}\right) \sigma_{q}}{\Lambda+k_{4}}+\frac{\left(1-q_{2}\right)}{\left(\Lambda+k_{5}\right)}\left(\frac{\eta_{i} \sigma_{i}}{\Lambda+k_{3}}+\frac{\eta_{q} \sigma_{q}}{\Lambda+k_{4}}\right)} .
$$

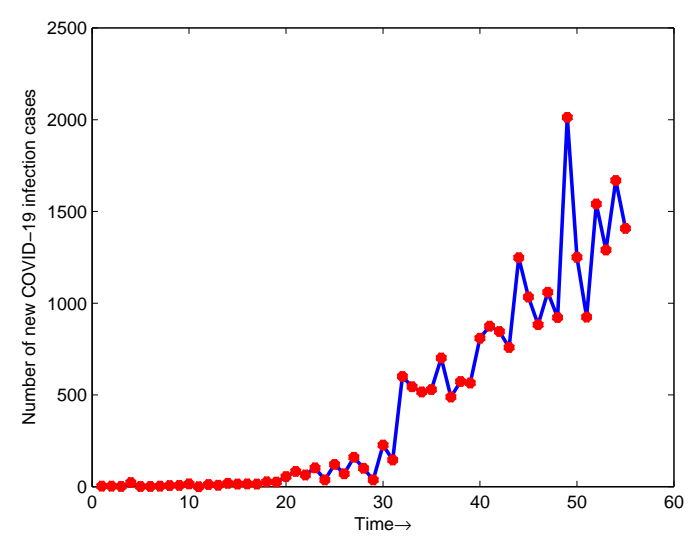

(a)

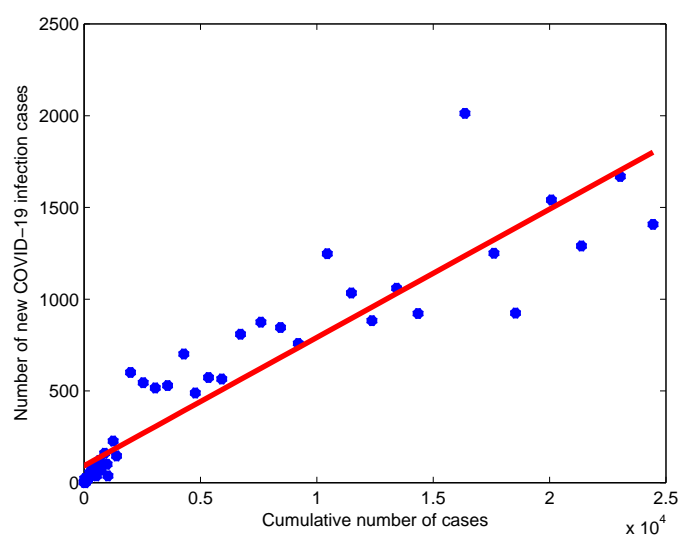

(b)

Figure 6: (a) Total outbreak size and (b) the daily number of cases against the cumulative number of cases.

In order to estimate the basic reproduction number $R_{0}$ from the expression (8), we have to estimate the force of infection $\Lambda$. According to [29], the daily number of new cases and the number of cumulative cases $q(t)$ are connected by the relation: the daily number of new cases $\sim \Lambda q(t)$. So, we can estimate $\Lambda$ by plotting the number of daily new cases verses the number of cumulative cases $q(t)$, the phase of exponential growth of the cumulative number of cases is evidenced by a linear growth of the curve, the 
slope of which is the force of infection $(\Lambda)$. This linear growth of the curve is computed by a leastsquare linear fit [29]. The reported data from $1^{\text {st }}$ March to $24^{\text {th }}$ April, 2020 for COVID-19 outbreak in India has been presented in Figure 6(a). Now on the basis of the slope of the line presented in Figure 6(b), we have $\Lambda=0.069929435282024 \pm 0.007427261807510$ day $^{-1}$. Using the expression (8) along with the above estimated $\Lambda$ and other parametric values presented in Table 2, we have the estimation of the basic reproduction number as $R_{0}=2.095744073$ with lower and upper values are 1.990274890 and 2.200561243 , respectively.

\subsection{Effective Reproduction Number}

We know that the basic reproduction number plays an important role in controlling the disease spreading. It is the average number of secondary infections during the infection period. Since when $R_{0}<1$ then the average number of secondary infections in the infection period is less than one and consequently the disease is easy to control. But as the number of secondary infections is changed with time for COVID-19 infection.

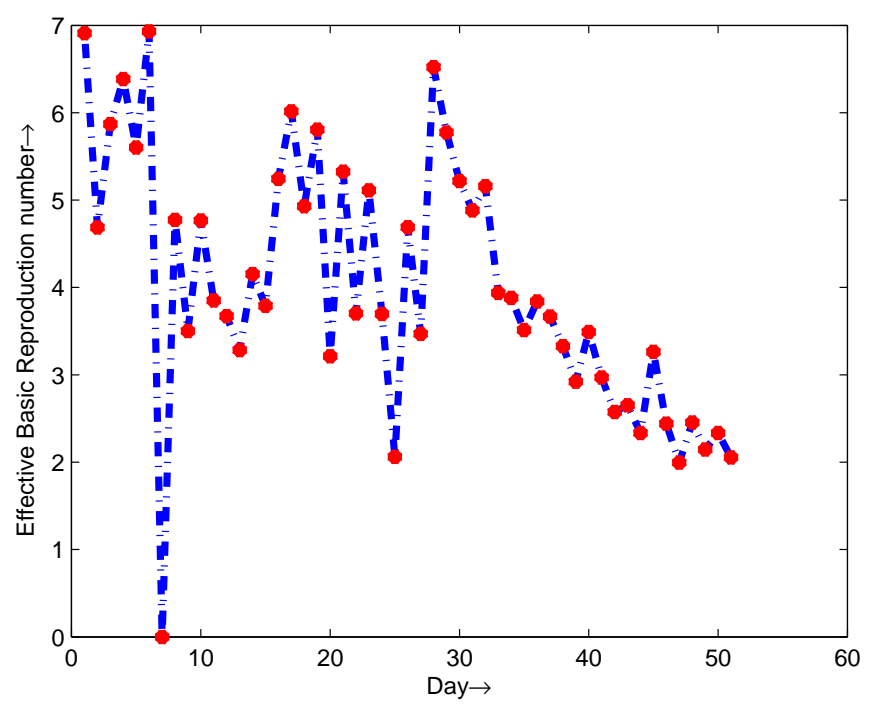

Figure 7: Effective reproduction number

In this section, we shall describe the effective reproduction number, denoted by $R(t)$, which is defined as the number of secondary infections by a single primary infection at the $t^{t h}$ day. Then the quantity $R(t)$ will give the information about the necessary steps to control the COVID-19 in India. The estimation of 
$R(t)$ can be done using the following renewal equation $[32,33,34]$

$$
R(t)=\frac{b(t)}{\int_{\tau=0}^{\infty} b(t-\tau) h(\tau) d \tau},
$$

where $b(t)$ is the number of new cases at $t^{t h}$ day and $h(\tau)$ is the generation interval distribution for the COVID-19 disease. It is the probability distribution function. Let the leaving rate of the infected class from the corresponding compartments be $m_{1}=\sigma_{a}+\sigma_{i}+\sigma_{q}+\mu, m_{2}=\gamma_{a}+\mu, m_{3}=\eta_{i}+\gamma_{i}+\mu+\delta$, $m_{4}=\eta_{q}+\gamma_{q}+\mu+\delta$ and $m_{5}=\gamma_{h}+\mu+\delta$. Therefore, the function will be the combination of the five exponential functions $m_{1} e^{-m_{1} t}, m_{2} e^{-m_{2} t}, m_{3} e^{-m_{3} t}, m_{4} e^{-m_{4} t}$ and $m_{5} e^{-m_{5} t}$ in the following form

$$
h(t)=\sum_{i=1}^{5} \frac{m_{1} m_{2} m_{3} m_{4} m_{5} e^{-m_{i} t}}{\prod_{j=1 j \neq i}^{5}\left(m_{j}-m_{i}\right)}
$$

with mean of the distribution is $T=\frac{1}{m_{1}}+\frac{1}{m_{2}}+\frac{1}{m_{3}}+\frac{1}{m_{4}}+\frac{1}{m_{5}}$ and $\tau>0$. The above relation is valid when the force of infection $\Lambda>\min \left\{-m_{1},-m_{2},-m_{3},-m_{4},-m_{5}\right\}$. Using the model estimated parameters, we have calculated the effective reproduction number that has been illustrated in the Figure 7. It is clear from the figure that the effective reproduction number oscillates but its value is above two in the considered interval except only one day. To control the disease we have to decrease its value and if we make it less than one then the disease will be controlled.

\section{Model Prediction and Some Preventive Measures}

In this section, we shall explore the model prediction in Indian aspect and seek the preventive measures to control COVID-19 in India. The model parameter $d$ denotes the proportion of population who maintain the social distancing from others (or stay at home in safe). According to our estimation $67.38 \%$ of the population maintain the social distancing from other people in the lock down period.

In Figure 8, we have presented the model predicted total outbreak size and the peak prevalence during the pandemic for different values of $d$ for the entire time period of prevalence. It is clear from the figures that if $80 \%$ people follow the lock down, then the disease is easy to control. Thus to control the disease, the administration should be strict to impose the lock down properly. As per our model predictions if only $20 \%$ or less people can not follow the lock down then disease may be controlled. So only the administrative, food and medicine supply persons may go outside in the lock down period. 


\subsection{Programme for Maintaining Safe Distancing and Successful Lock-down}

A part of population maintain social distancing or safe distancing to protect the virus transmission. In the model, this part or fraction is denoted by the parameter $d$. In order to find the possible impact of the parameter $d$ on the total outbreak and peak prevalence, we have plotted total outbreak with respect to $d$ in Figure 8(a) and the peak prevalence with respect to $d$ in Figure 8(b). According to our model, the total outbreak size $(q(t, \Phi))$ is governed by the relation (5). Our findings show that due to increase of $d$ (proportion of population who maintain social distancing) upto 0.77 , the total outbreak size reduces from $100 \%$ to $13 \%$ (Fig. 8 (a)) and the peak prevalence reduces from $37.77 \%$ to $0.28 \%$ i.e at a negligible level (Fig. 8(b)).

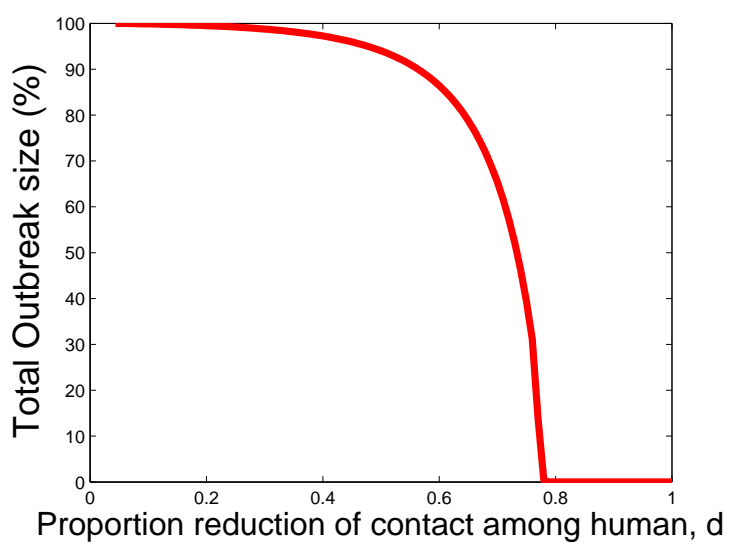

(a)

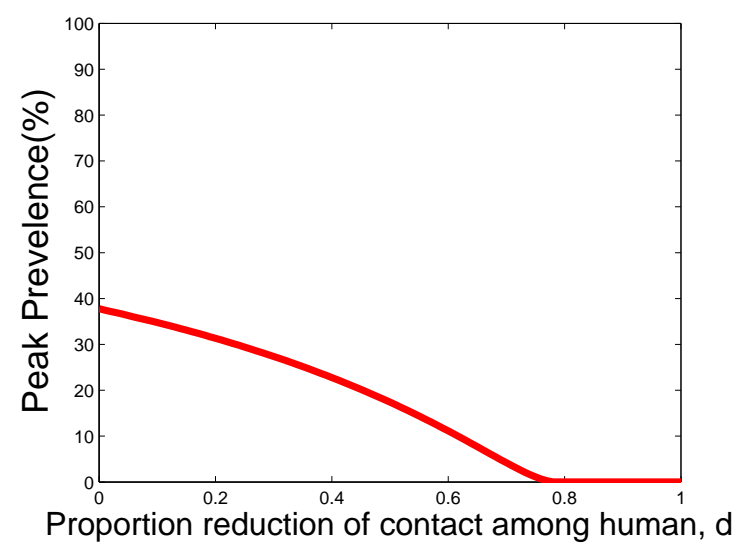

(b)

Figure 8: (a) Total outbreak size and (b) Peak prevalence during the pandemic predicted by the model versus the lock-down parameter $\mathrm{d}$.

The parameter $d$ can be increased by awareness programmes through public media like T.V. programmes, mobile messages or broadcasting through any local media. The informations regarding the infection like number of infected population, number of COVID-19 related death in the local areas should be provided in proper time regularly and repeatedly. Such informations will increase the consciousness of the people in the affected areas and they will maintain the social distancing from each other and increase the value of $d$. On the other hand, the wrong informations can misguide the people and as a result $d$ will be decreased. India is a high population density country. Many peoples gather in market, ration shop to collect different food items and they do not maintain minimum distance from each other as a result $d$ decreases. In 
order to solve this problem self help group can be used for home delivery of different food items and other necessary requirements with expenses or without expenses (for BPL citizen). Formation of self help group and their instant training in each local areas can be done with the help of local administration (panchayat, municipality corporation, etc). In this way the supply of necessary requirements can be done in a systematic way and the parameter $d$ can be increased in a significant amount even after lock-down period.

Another notable factor is that a major portion Indian use the public vehicles like train, bus and air transport. It is impossible to maintain safe distancing among such vehicles passengers and so the parameter $d$ will decrease. A susceptible may be infected by an infectious person when they travel in same vehicle without maintaining the safe distancing. In this way the virus infection spreads from infected areas (red zone) to uninfected areas (green zone). So, our proposal is that the public vehicles should be closed in entire pandemic period.

\subsection{Prevention Measures on Asymptomatic Class}

Due to high population density, in general, the human to human contact rate is high in India. On the other hand, an asymptomatic infected human has no symptom but he/ she is able to transmit the virus to other susceptible human. So susceptible person will not take or take in late (after diagnostic test) prevention measure to protect from the virus transmission from an asymptomatic infected person. In this context our finding shows that approximately $45 \%$ of the infected population are asymptomatic. The time series of different infected populations (see Figure 5(b)) show that the number of daily asymptomatic infected population is larger than other infected population. The contribution on basic reproduction number of asymptomatic infected class is larger than other compartment. So protection of the virus transmission from asymptomatic infected class is a challenging problem for highly populated country like India.

In order to solve this problem, we propose a prevention policy that reduces contact rate between asymptomatic infected human and susceptible human. In this context first of all we have to identify the asymptomatic infected human among the population and then keep them in quarantine. To identify asymptomatic infected human, we have to focus on the diagnostic test among major portion of population, specially in the affected areas and then they should be quarantined.

Let $p$ with $0 \leq p \leq 1$ be an effectiveness of this prevention policy ( that is the reduction of contact rate among asymptomatic infected human and susceptible human ), applying such policy causes the follow- 
ing transformation in the model: $\rho \rightarrow(1-p) \rho$. The impact of such prevention $(p)$ on total outbreak size has been illustrated in Figure 9(a) and impact on peak prevalence has been presented in Figure 9(b). Our findings suggest that enhancing $p$ by at least $72 \%$ the the total outbreak size will be reduced from $94.65 \%$ to nearly $2.772 \%$ whereas the peak prevalence reduces by $18 \%$.

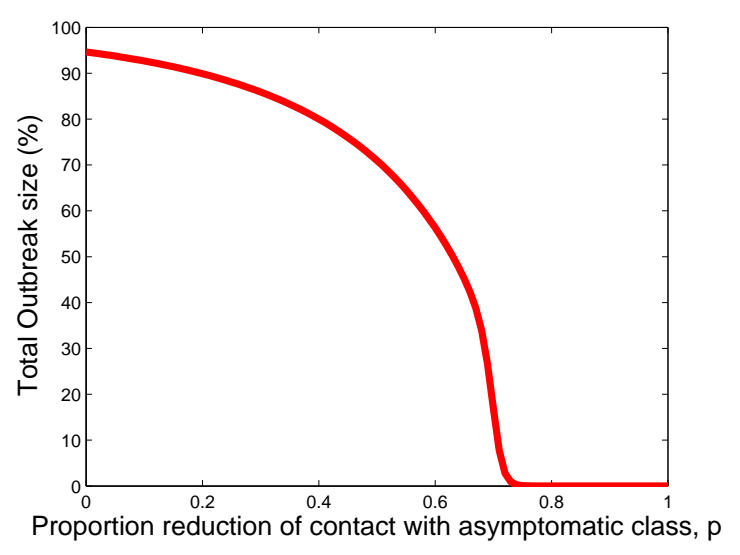

(a)

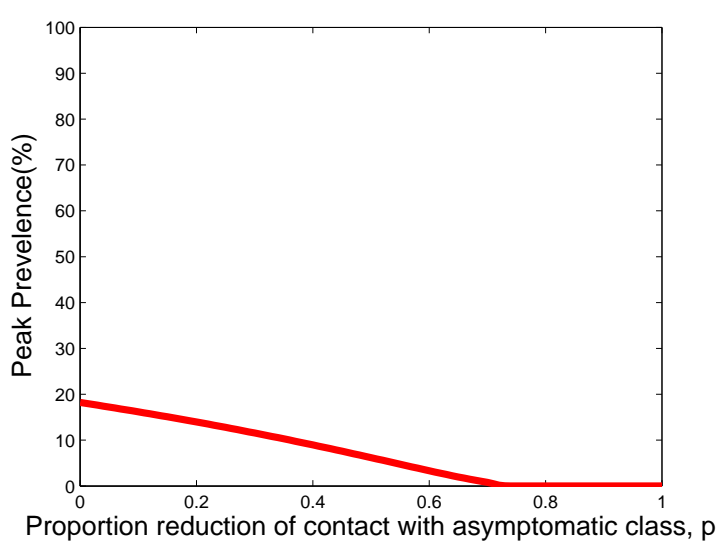

(b)

Figure 9: (a) Total outbreak size and (b) Peak prevalence during the pandemic predicted by the model versus the parameter $p$.

\subsection{Prevention Programme on Quarantine, Isolation and Rapid Hospitalisation}

In the real field of Indian scenario, we have observed that many staffs of quarantine and isolation centres (hospitals) have been infected by the quarantined and isolated infected persons. In this model, $q_{1}$ and $q_{2}$ represent the effectiveness of quarantine and isolation, respectively. For perfect quarantine and isolation $q_{1}=1$ and $q_{2}=1$, but real information suggests that $q_{1}<1, q_{2}<1$. So, we have to focus on perfect quarantine and isolation and our model suggests that by increasing $q_{1}$ from 0.94 to 1 and $q_{2}$ from 0.94 to 1 simultaneously, the total outbreak size reduces by $2.19 \%$ and this fact has been illustrated in Figure 10(a). Our another observation is that according to our estimation the rate of hospitalisation from non-quarantined infected $\left(\eta_{i}\right)$ and quarantined infected classes $\eta_{q}$ are 0.26 and 0.40 , respectively. We have to increase these rates to control the COVID-19 outbreak. If we increase these rates up to 0.5 and 1 , respectively then the total number of infected population will be decreased by $2 \%$ whose graphical presentation has been given in Figure 10(b). By both the above said preventions the outbreak can also be delayed. 


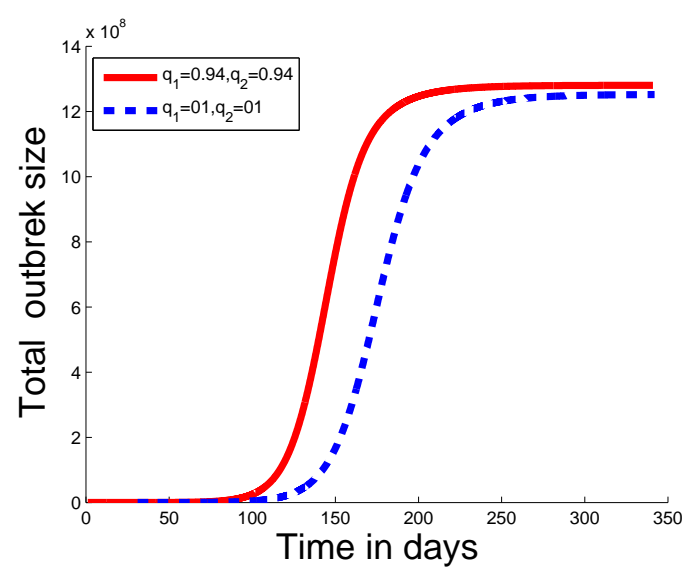

(a)

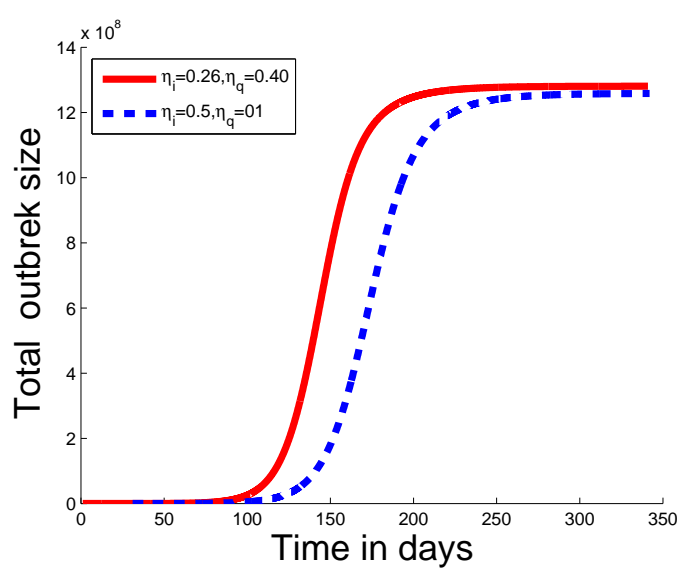

(b)

Figure 10: Time series of total outbreak size for (a) original parameter (solid line) and increased values (dash line) of $q_{1}$ and $q_{2}$ and (b) original parameter (solid line) and increased values (dash line) of $\eta_{i}$ and $\eta_{q}$.

In order to control this outbreak, we have to apply above said prevention programmes simultaneously. In this case we increase the values of parameters $d, p, q_{1}, q_{2}, \eta_{i}$ and $\eta_{q}$ from the estimated values (Table 3 ) to the values $d=0.2, p=0.5, q_{1}=1, q_{2}=1 \eta_{i}=0.5, \eta_{q}=1$ and the other parameters remain same (Table 3). Now using the above changed parametric values, we continue the time series of cumulative number of infected population from $24^{\text {th }}$ April, 2020 for the next 311 days along with the previous series (See Figure 11(a)). In the figure, red solid line denotes the real scenario (generates from the estimated parametric values) for the time period $25^{\text {th }}$ March to $24^{\text {th }}$ April, 2020 and the dash blue line represents the proposed time series generated due to control. Under the above control the number of cumulative infected population will be $4.194 \times 10^{4}$ and the disease will die out after a short time period. The effect of the above said control on the various infected classes are illustrated in Figure 11(b), here solid line denotes the time series for the estimated parameter for the first 31 days after implement of lock-down and then we apply the control and the corresponding time series is denoted by dash line. Under the above control the prevalence will be maximum around on $27^{\text {th }}$ April, 2020 and the disease will die out around on $31^{\text {th }}$ August, 2020. 


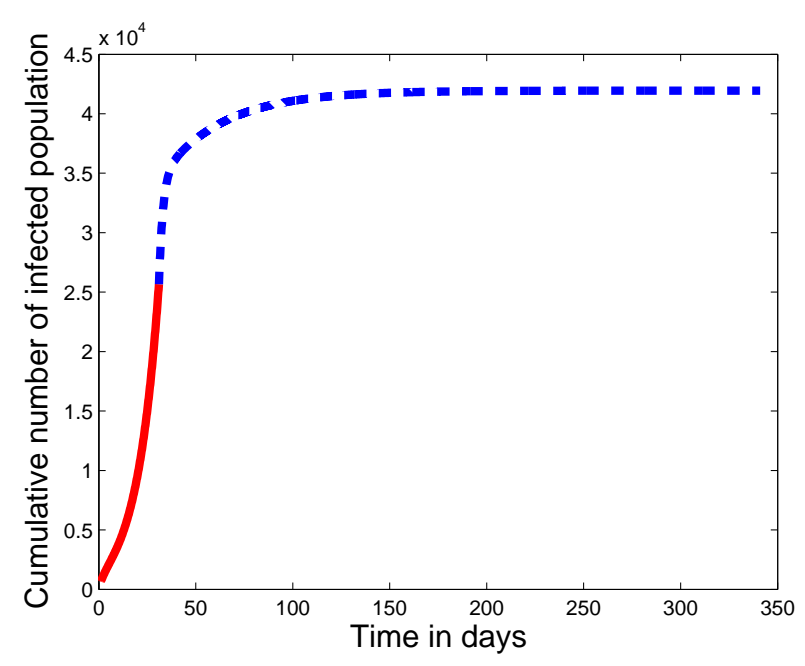

(a)

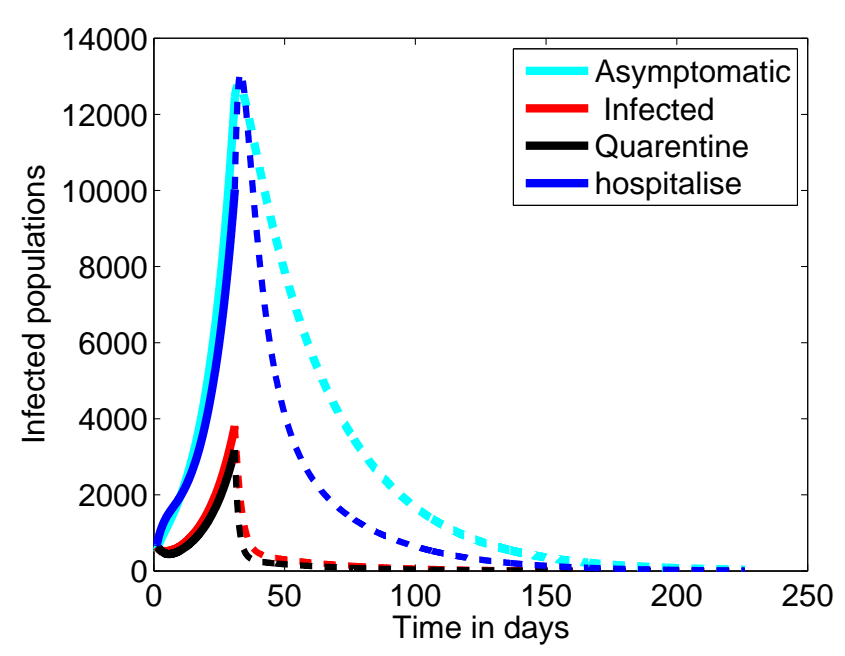

(b)

Figure 11: Time series for (a) cumulative number of infected population due to control applied from $25^{\text {th }}$ April, 2020 (blue dash line), (b) time series of infected classes due to control applied from $25^{\text {th }}$ April, 2020 (dash line).

\section{Conclusions}

In this research work, we have formulated a deterministic compartmental model to study the dynamics and future trend of COVID-19 outbreak in India and to give prediction on the future outbreak. We have studied the basic properties of the model and obtained the expression of the basic reproduction number and different contributory parts by infected classes. We investigate the local stability analysis of the disease free equilibrium point, which is locally asymptotically stable for $R_{0}<1$ and unstable for $R_{0}>1$.

To fit the proposed model to the reported cumulative data of COVID-19 outbreak in India, we have estimated the model parameters using the real cases of COVID-19 in India. In this connection we consider two sets of data. The first set contains the cases reported during the period $1^{\text {st }}$ March to $24^{\text {th }}$ April, 2020. On the other hand before the implementation of lock-down (25 $5^{\text {th }}$ March, 2020) many symptomatic as well as asymptomatic infected person came in India from other COVID-19 pandemic countries who are neither detected nor reported properly. So there remains uncertainty in the data, reported during the period $1^{\text {st }}$ March to $24^{\text {th }}$ March, 2020. Thus, we consider the second set of data, reported during the period $25^{\text {th }}$ March to $24^{\text {th }}$ April, 2020. We have fitted the model to the both sets of data and es- 
timated two sets of parameters. By using the estimated parametric values (from set-2), we compute the average basic reproduction number which is $R_{0}=2.414190966$ among which the contributions of asymptomatic, symptomatic non-quarantined, symptomatic \& quarantined, hospitalised infected classes are $R_{0 A}=1.842473415, R_{0 I}=0.3910030945, R_{0 Q}=0.01860012179, R_{0 H}=0.1621143316$, respectively. According to sensitivity analysis the virus transmission rate $(\beta)$, lock-down effect $(d)$, ratio of transmission rates of asymptomatic class and symptomatic class $(\rho)$, recovery rate from asymptomatic class $\left(\gamma_{a}\right)$ are the most influential parameters. The basic reproduction number calculated from actual data lies between 1.99 to 2.20 . The study of the effective basic reproduction number shows that it declines from 6.91 to 1.995 .

Our findings show that the outbreak size and peak prevalence can be reduced from $100 \%$ to $13 \%$ and from $37.77 \%$ to $0.28 \%$, respectively by increasing the proportion of the people who maintain the safe distancing from each other $(d)$ by 0.77 . The findings show that the increasing of the proportion of asymptomatic infected population detected and isolated $(p)$ by at least $72 \%$ causes the reduction of the total outbreak size from $94.65 \%$ to nearly $2.772 \%$ whereas the peak prevalence reduces by $18 \%$.

Our findings also suggest that the dramatical reduction in total outbreak size and peak prevalence are possible by increasing $d$ (the proportion of number of population who maintain safe distancing from each other) to a certain level and $p$ (the proportion of number of asymptomatic population who are detected and isolated from the other susceptible population) to a certain level. To control the disease, we also have to increase the detection (on the basis of diagnostic test) and hospitalized rates $\left(\eta_{i}\right.$ and $\left.\eta_{q}\right)$ to a certain level.

\section{References}

[1] World Health Organization, Pneumonia of Unknown Cause-China, 2020. Available from: https: //www.who.int/csr/don/05-january-2020-pneumonia-of-unkown-cause-china/en/.

[2] World Health Organization. Updated WHO advice for international traffic in relation to the outbreak of the COVID-19. Geneva: WHO 2020 ; Available from: https://www.who.int/ith/COVID19 advice for international traffic /en/, accessed on April 11, 2020 .

[3] World Health Organization, Clinical Management of Severe Acute Respiratory Infection When Infection is Suspected, 2020. Available from: https://www.who.int/publications-detail/ clinical- 
management-of-severe-acute-respiratory-infection-when-novel-/ coronavirus-(ncov)-infection-issuspected.

[4] Event Horizon - COVID-19. Coronavirus COVID=19 Global Risk Assessment. Available from: http://rocs.hu-berlin.de/ corona/ relative-import-risk, accessed on February 26, 2020.

[5] Q. Li, X. Guan, P. Wu, X. Wang, L. Zhou, Y. Tong, et al., Early transmission dynamics in Wuhan,China, of novel coronavirus-infected pneumonia, N. Engl. J. Med., (2020).

[6] Chowell, G., Fenimore, P. W., Castillo-Garsow, M. A. et al., SARS outbreak in Ontario, Hong Kong and Singapore: the role of diagnosis and isolation as a control mechanism, Los Alamos Unclassified Report LA-UR-03-2653, 2003.

[7] Martcheva, M.: An Introduction to Mathematical Epidemiology. Springer, New York (2015)

[8] Brauer, F. and Castillo-Chavez, C. (2001). Mathematical Models in Population Biology and Epidemiology, Texts in Applied Math. 40, Springer-Verlag, New York.

[9] Z. Ma and J. Li, Dynamical Modeling and Anaylsis of Epidemics,, World Scientific, (2009) .

[10] World Health Organization, Middle East respiratory syndrome coronavirus (MERS-CoV), 2019, https://www.who.int/news-room/fact-sheets/detail/middle-east-respiratory-syndromecoronavirus-(mers-cov).

[11] J Guarner, Three Emerging Coronaviruses in Two Decades The Story of SARS, MERS, and Now COVID-19, Am J Clin Pathol. 2020 Mar; 153(4): 420-421.

[12] D. S. Hui, E. I. Azhar, T. A. Madani, F. Ntoumi, R. Kock, O. Dar, et al., The continuing 2019nCoV epidemic threat of novel coronaviruses to global health-The latest 2019 novel coronavirus outbreak in Wuhan, China, Int. J. Infect. Dis., 91 (2020), 264-266.

[13] Wu JT, Leung K, Bushman M, Kishore N, Niehus R, de Salazar PM, et al. Estimating clinical severity of COVID-19 from the transmission dynamics in Wuhan, China. Nature Medicine. (2020). DOI: 10.1038/s41591-020-0822-7.[20] 
[14] Wu JT, Leung K, Leung GM. Nowcasting and forecasting the potential domestic and international spread of the 2019-nCoV outbreak originating in Wuhan, China: A modelling study. Lancet 2020; 395 : 689-97.

[15] X. Rong, L. Yang, H. Chu, M. Fan, Effect of delay in diagnosis on transmission of COVID-19, Mathematical Bioscience and Engineering, 2020. 17(3): 2725-2740.

[16] National Health Commission of the People's Republic of China, Situation Report of the Pneumonia Cases Caused by the Novel Coronavirus, 2020. Available from: http://www.nhc.gov.cn/xcs/ yqfkdt/202002/3db09278e3034f289841300ed09bd0e1.shtml.

[17] C. Huang, Y.Wang, X. Li, L. Ren, J. Zhao, Y. Hu, et al., Clinical features of patients infected with 2019 novel coronavirus in Wuhan, China, Lancet, 395 (2020), 497-506.

[18] Wu JT, Leung K, Leung GM. Nowcasting and forecasting the potential domestic and international spread of the 2019-nCoV outbreak originating in Wuhan, China: a modelling study. Lancet 2020; Available from: http://www.sciencedirect.com/science/article/pii/S1201971220300539.

[19] Bogoch II, Watts A, Thomas-Bachli A, Huber C, Kraemer MUG, Khan K. Pneumonia of unknown etiology in Wuhan, China: potential for international spread via commercial air travel.J Travel Med 2020;, doi:http://dx.doi.org/10.1056/ NEJMoa2002032.

[20] National Centre for Disease Control. Travel Advisory. 5 February, 2020. Available from: https://ncdc.gov.in/ WriteReadData/1892s/63950984511580999086.pdf., accessed on February $16,2020$.

[21] Worldometer, COVID-19 CORONAVIRUS PANDEMIC, 2020, https://www.worldometers.info/coronavirus/\#countries.

[22] Q Lin, S Zhao, D Gao, Y Lou, S Yang. "A conceptual model for the coronavirus disease 2019 (COVID-19) outbreak in Wuhan, China with individual reaction and governmental action.” International journal of infectious diseases (2020).

[23] National Centre for Disease Control. COVID-19 outbreak in China - Travel advisory to travelers visiting China. Available from: https://ncdc.gov.in/WriteReadData/ 1892s/34827556791580715701.pdf, accessed on February 11, 1. 2020. 
[24] Hethcote H W. The mathematics of infectious diseases. SIAM Rev.2000; 42(4). 599-653.

[25] Diekmann, O., Heesterbeek, J. A. P., and Metz, J. A. J. (1990). On the definition and the computation of the basic reproduction ratio $R_{0}$ in the models for infectious disease in heterogeneous populations.J Math Biol 28: 365-382.

[26] Van den Driessche, P., Watmough, J.: Reproduction Numbers and Sub-Threshold Endemic Equilibria for Compartmental Models of Disease Trans-mission. Math Biosci. 180, 29-48 (2002)

[27] Chitnis, N., Hyman, J. M., Cushing, J. M. (2008). Determining important parameters in the spread of malaria through the sensitivity analysis of a mathematical model. Bulletin of Mathematical Biology, 70(5), 1272-1296.

[28] S.K. Biswas,U. Ghosh, S. Sarkar, Mathematical model of zika virus dynamics with vector control and sensitivity analysis.Infectious Disease Modelling 5 (2020) 23-41.

[29] Favier C. (2006) Early determination of the reproductive number of vector-borne diseases: the case of dengue in Brazil. Trop. Med. Int. Health 11, 332-340.

[30] Massad E.,Coutinho F.A.B, Burattini M.N, Amaku M. (2010): Estimation of $R_{0}$ from the initial phase of an outbreak of a vector-borne infection., Trop. Med. Int. Health 15(1) 2010 120-26.

[31] Massad E.,Coutinho F.A.B, Burattini M.N, Lopez L.F. (2001) The risk of yellow fever in a dengueinfested area. Trans. R. Soc. Trop. Med. Hyg. 95 370-374.

[32] J.Wallinga, M. Lipsitch, How generation intervals shape the relationship between growth rates and reproductive numbers., Proc. R. Soc. B 274 (2007) 599-604.

[33] S. Pinho, C. Ferreira, L. Esteva, F. Barreto, V. Morato e Silva, et al., Modelling the dynamics of dengue real epidemics., Phil. Trans. R. Soc. A 368 (2010) 5679-92.

[34] T. Sardar, S. Rana, S. Bhattacharya, K. Khaled, J. Chattopadhyay, A generic model for a single strain mosquito-transmitted disease with memory on the host and the vector, Math. Biosci. 263 (2015) 18-36.

[35] "SOUTH ASIA::INDIA".CIA.gov, Central Intelligence Agency Retrieved 7 February 2020. 